\title{
Recent results from JLab on nucleon responses
}

Kees de Jager

Jefferson Laboratory, Newport News, VA 23606, USA

Received: 9 Sep 2003 / Accepted: 14 Nov 2003 /

Published Online: 6 Feb 2004 - (C) Società Italiana di Fisica / Springer-Verlag 2004

\begin{abstract}
Highlights of recent data from Jefferson Lab on the nucleon response to an electro-magnetic probe are presented. Recent technological advances in polarized beams and either polarized targets or nucleon recoil polarimeters have yielded a significant improvement on the precision of the data. An outlook is presented of planned experiments.
\end{abstract}

PACS. 24.85.+p - 25.30.-c

\section{Electro-magnetic form factors}

\subsection{Introduction}

The nucleon electromagnetic form factors (EMFF) are of fundamental importance for the understanding of the nucleon's internal structure. In Plane Wave Born Approximation (PWBA) the cross section for elastic electronnucleon scattering can be expressed in terms of the Dirac and Pauli form factors $F_{1}$ and $F_{2}$, respectively,

$\frac{d \sigma}{d \Omega}=\sigma_{M}\left[\left(F_{1}^{2}+\kappa^{2} \tau F_{2}^{2}\right) \cos ^{2}\left(\frac{\theta_{e}}{2}\right)+2 \tau\left(F_{1}^{2}+\kappa F_{2}^{2}\right)^{2} \sin ^{2}\left(\frac{\theta_{e}}{2}\right)\right]$

where $\tau=Q^{2} / 4\left(m_{N}^{2}\right), Q$ is the four-momentum transfer, $m_{N}$ the mass of the nucleon, $\sigma_{M}$ the Mott cross section for scattering off a point-like particle, $\kappa$ the nucleon anomalous magnetic moment, $\theta_{e}$ the electron scattering angle and $E_{e}$ the electron energy. $F_{1}$ and $F_{2}$ can be expressed in the electric and magnetic Sachs form factors $G_{E}$ and $G_{M}$, respectively,

$$
\begin{gathered}
G_{E}=F_{1}-\tau \kappa F_{2} \\
G_{M}=F_{1}+\kappa F_{2}
\end{gathered}
$$

leading to the so-called Rosenbluth [1] formula

$$
\frac{d \sigma}{d \Omega}=\sigma_{M}\left[\frac{G_{E}^{2}+\tau G_{M}^{2}}{1+\tau}+2 \tau G_{M}^{2} \tan ^{2}\left(\frac{\theta_{e}}{2}\right)\right]
$$

This equation illustrates that $G_{E}$ and $G_{M}$ can be determined separately by performing cross-section measurements at fixed $Q^{2}$ over a range of $\left(\theta_{e}, E_{e}\right)$ combinations (Rosenbluth separation). In the non-relativistic Breit frame the Sachs form factors can be identified with the Fourier transform of the nucleon charge and magnetization density distributions.
Through the middle of the previous decade practically all available proton EMFF data had been collected using the Rosenbluth separation technique. This experimental procedure requires an accurate knowledge of the electron energy and the total luminosity. In addition, since the contribution to the elastic cross section from the magnetic form factor is weighted with $Q^{2}$, data on $G_{E}^{p}$ suffer from increasing systematic uncertainties at higher $Q^{2}$-values. The then available world data set [2] was compared to the so-called dipole parametrization $G_{D}$, which corresponds to exponentially decreasing radial charge and magnetization densities:

$$
G_{D}=\left(\frac{\Lambda^{2}}{\Lambda^{2}+Q^{2}}\right)^{2} \text { with } \Lambda=0.84 \mathrm{GeV} / \mathrm{c} \text { and } Q \text { in } \mathrm{GeV} / \mathrm{c}
$$

For all four EMFF the available data agreed with the dipole parametrization to within $20 \%$. Both the $G_{E}^{p}$ and the $G_{M}^{p}$ data could be fitted adequately with an identical parametrization. However, the limitation of the Rosenbluth separation was evident from the fact that different data sets for $G_{E}^{p}$ scattered by up to $50 \%$ at higher $Q^{2}$ values.

\subsection{Polarization instrumentation}

Over 20 years ago Akhiezer and Rekalo [3] and Arnold et al. (4) showed that the accuracy of EMFF measurements could be increased significantly by scattering polarized electrons off a polarized target (or equivalently by measuring the polarization of the recoiling nucleon). Technological advances have only recently made possible a large number of new data with a significantly improved accuracy. Polarized electron beams [5]6] are now reliably available with a polarization close to $80 \%$ at currents of up to $100 \mu \mathrm{A}$. The beam polarization is measured with either Møller 7] or Compton [8] polarimeters with an accuracy 
approaching $1 \%$. The dynamical polarization technique $[9]$ provides polarized hydrogen or deuterium targets with an average polarization of 80 or $20 \%$, respectively, while polarized helium targets are available with a polarization close to $50 \%$, either through spin [10] or metastability [11] exchange at a density of $10 \mathrm{~atm}$. Finally, the polarization of recoiling or knocked-out reaction products can be measured with focal-plane [12] or neutron [13] polarimeters.

\subsection{Theory}

A frequently used framework to describe the EMFF is that of Vector Meson Dominance (VMD) [14, in which one assumes that the virtual photon - after having become a quark-antiquark pair - couples to the nucleon as a vector meson. The EMFF can then be expressed in terms of coupling strengths between the virtual photon and the vector meson and between the vector meson and the nucleon, summing over all possible vector mesons. A common restriction of VMD models is that they do not predict a correct behaviour of the EMFF at high $Q^{2}$-values. Lomon 15 constructed a hybrid model which couples the low $Q^{2}$ behaviour of the VMD model to the asymptotic behaviour predicted by pQCD.

Several models [16,17, 18 have incorporated constituent quarks on the light front. In doing this the pQCD condition that the transverse momentum is zero, is freed and an orbital angular momentum component is introduced in the wavefunction of the proton, thus giving up helicity conservation. All these models predict a linear dropoff of $G_{E}^{p} / G_{M}^{p}$ with $Q^{2}$, or equivalently $F_{2} / F_{1} \propto 1 / Q$.

However, all presently available theories are at least to some extent effective (or parametrizations). Only lattice gauge theory can provide a truely ab initio calculation, but accurate lattice QCD results for the EMFF are still several years away.

\subsection{Neutron magnetic form factor}

Significant progress has been made in measurements of $G_{M}^{n}$ at low $Q^{2}$-values by measuring the ratio of quasielastic neutron and proton knock-out from a deuterium target. This method is insensitive to nuclear binding effects and to fluctuations in the luminosity and detector acceptance. The basic set-up used in all such measurements is very similar: the electron is detected in a magnetic spectrometer with coincident neutron/proton detection in a large scintillator array. The main technical difficulty in such a ratio measurement is the absolute determination of the neutron detection efficiency. Recently, inclusive quasielastic scattering of polarized electrons off a polarized ${ }^{3} \mathrm{He}$ target was measured 27] in Hall A at JLab in a $Q^{2}$-range from 0.1 to $0.6(\mathrm{GeV} / \mathrm{c})^{2}$. This experiment has provided an independent accurate measurement of $G_{M}^{n}$ at $Q^{2}$-values of 0.1 and $0.2(\mathrm{GeV} / \mathrm{c})^{2}$, in excellent agreement with the Mainz data. At the higher $Q^{2}$-values $G_{M}^{n}$ was extracted 28 in PWIA, since final-state-interaction effects are expected to decrease with increasing $Q^{2}$. A study of $G_{M}^{n}$ at

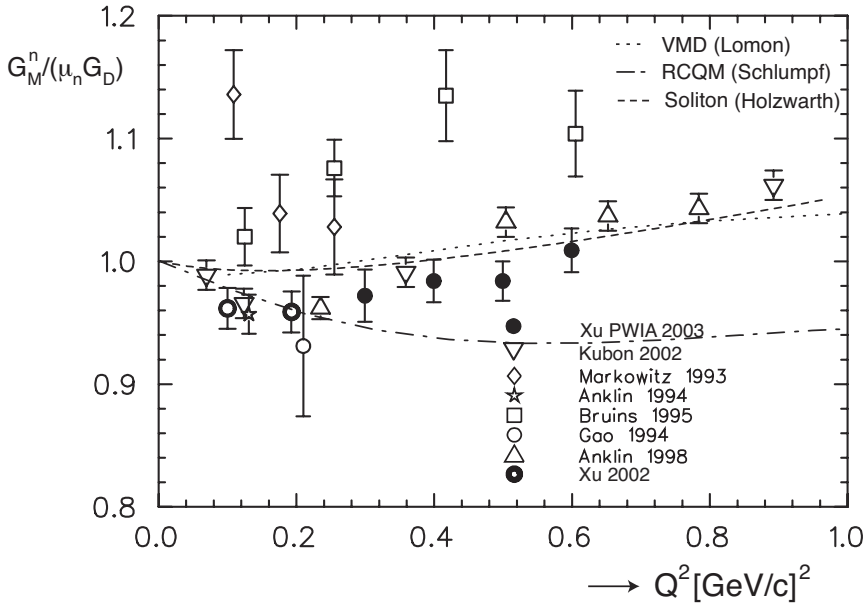

Fig. 1. The neutron magnetic form factor $G_{M}^{n}$, in units of $\mu G_{D}$, as a function of $Q^{2}$. The data are from 21 22, 23, 24 25 26, 27 28]. The theory curves are: dotted [15, dot-dashed [16. and short-dashed 20]

$Q^{2}$-values up to $5(\mathrm{GeV} / \mathrm{c})^{2}$ has recently been completed in Hall $\mathrm{B}$ by measuring the neutron/proton quasi-elastic cross-section ratio using the CLAS detector 29.

Figure 1 1 shows the results of all completed $G_{M}^{n}$ experiments. The Mainz $G_{M}^{n}$ data are 8-10\% lower than those from ELSA, at variance with the quoted uncertainty of appr. $2 \%$. This discrepancy would require a $16-20 \%$ error in the detector efficiency. Two of the theoretical predictions shown [15,20] provide an accurate description of the data.

\subsection{Neutron electric form factor}

In the last decade a series of spin-dependent measurements have provided accurate data on $G_{E}^{n}$ by utilizing the fact that the ratio of the beam-target asymmetry with the target polarization perpendicular and parallel to the momentum transfer is directly proportional to the ratio of the electric and magnetic form factors. A similar result is obtained with the reaction ${ }^{2} \mathrm{H}\left(\mathbf{e}, e^{\prime} \mathbf{n}\right)$ when one measures the polarization of the recoiling neutron directly and after having precessed the neutron spin over $90^{\circ}$ with a dipole magnet.

Figure 2 shows results obtained through the reactions ${ }^{2} \mathrm{H}\left(\mathbf{e}, e^{\prime} n\right)$ 32, ${ }^{2} \mathrm{H}\left(\mathbf{e}, e^{\prime} \mathbf{n}\right)\left[3,33\right.$ and ${ }^{3} \overrightarrow{\mathrm{He}}\left(\mathbf{e}, e^{\prime} n\right)[34$, 35. At low $Q^{2}$-values corrections for nuclear medium and rescattering effects can be sizeable: $65 \%$ for ${ }^{2} \mathrm{H}$ at 0.15 $(\mathrm{GeV} / \mathrm{c})^{2}$ and $50 \%$ for ${ }^{3} \overrightarrow{\mathrm{He}}$ at $0.35(\mathrm{GeV} / \mathrm{c})^{2}$. These corrections are expected to decrease significantly with increasing $Q$, although no reliable calculations are presently available for ${ }^{3} \overrightarrow{\mathrm{He}}$ above $0.5(\mathrm{GeV} / \mathrm{c})^{2}$. Thus, there are now data from a variety of reaction channels available in a $Q^{2}$ range up to $0.6(\mathrm{GeV} / \mathrm{c})^{2}$ with an overall accuracy of appr. $20 \%$, which are in mutual agreement. The prediction by Miller et al. [17, using a constituent quark model on the light front, is in good agreement with the sofar available data, with the pion cloud dominating at low $Q^{2}$-values. 

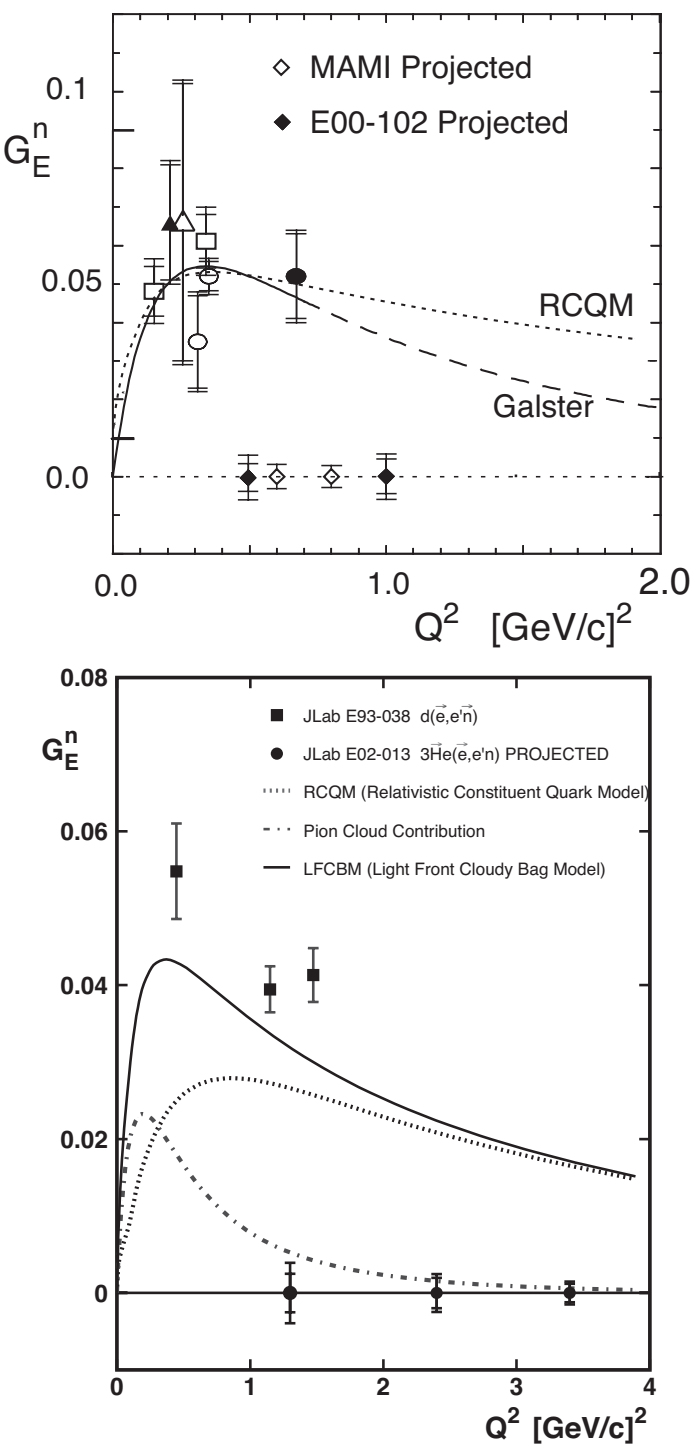

Fig. 2. The neutron electric form factor $G_{E}^{n}$ as a function of $Q^{2}$. The data are indicated by the following symbols: solid: triangle 32, squares [39, diamonds [38, and circle 35]; open: circles 3134], squares [13 33], triangle [30] and diamonds 37]. The curves represent Miller's RCQM prediction 17 (dotted) and the Galster parametrization [36] (full/dashed). The bottom plot shows the projected accuracy of E02-013 [41] and the separate contributions to the prediction of Miller. Symbols on the zero axis indicate the $Q^{2}$-value and the size of the error bars of ongoing experiments

The 30 years old Galster parametrization [36] provides a fortuitously good description of the data.

Shown on the horizontal axis in Fig. [2] are results expected in the near future, from the ${ }^{2} \mathrm{H}\left(\mathbf{e}, e^{\prime} n\right) 38$ channel at JLab and from the ${ }^{2} \mathrm{H}\left(\mathbf{e}, e^{\prime} \mathbf{n}\right) 37$ channel at Mainz. In the bottom plot the results for the ${ }^{2} \mathrm{H}\left(\mathbf{e}, e^{\prime} \mathbf{n}\right)$ channel 39 are shown which extend the existing data set to 1.5 $(\mathrm{GeV} / \mathrm{c})^{2}$ with great accuracy. Also shown is the pioncloud contribution which Miller added phenomenologically to his RCQM model in order to obtain agreement with the data. Recently an experiment 41] has been ap- proved at JLab to measure $G_{E}^{n}$ up to a $Q^{2}$-value of 3.4 $(\mathrm{GeV} / \mathrm{c})^{2}$ using the ${ }^{3} \overrightarrow{\mathrm{He}}\left(\mathbf{e}, e^{\prime} n\right)$ reaction. In addition, the BLAST facility 40] at MIT is expected to provide accurate data on $G_{E}^{n}$ in a $Q^{2}$-range from 0.1 to $0.8(\mathrm{GeV} / \mathrm{c})^{2}$. Thus, within a couple of years $G_{E}^{n}$ data with an accuracy of $10 \%$ or better will be available up to a $Q^{2}$-value of 3.4 $(\mathrm{GeV} / \mathrm{c})^{2}$.

\subsection{Proton electric form factor}

The polarization-transfer technique has recently been used in two experiments 42,43 in Hall A at JLab, to measure the ratio $G_{E}^{p} / G_{M}^{p}$ in a $Q^{2}$-range from 0.5 to $5.6(\mathrm{GeV} / \mathrm{c})^{2}$. Elastic ep events were selected by detecting electrons and protons in coincidence in the two identical HRS spectrometers. At the four highest $Q^{2}$-values a lead-glass calorimeter was used to detect the scattered electrons. The polarization of the recoiling proton was determined with a Focal Plane Polarimeter (FPP) in the hadron HRS, consisting of two pairs of straw chambers with a carbon or polyethylene analyzer in between. The data were analyzed in bins of each of the target coordinates. No dependence on any of these variables was observed. The results for the ratio $G_{E}^{p} / G_{M}^{p}$ are shown in Fig. 3. The most striking feature of the data is the sharp decline as $Q^{2}$ increases. Since it is known that $G_{M}^{p}$ closely follows the dipole parametrization, it follows that $G_{E}^{p}$ falls more rapidly with $Q^{2}$ than $G_{D}$. A comparison with the older data from SLAC confirms the expected improvement in accuracy of such a spin-dependent measurement. All theoretical calculations shown in Fig. 3 predict a gradual decrease of $G_{E}^{p}$. At the highest $Q^{2}$-values the data do not follow pQCD scaling [45], but rather the $1 / Q$ behaviour in $F_{2} / F_{1}$, predicted by RCQM models. If one assumes the linear decrease in $G_{E}^{p} / G_{M}^{p}$ to continue, $G_{E}^{p}$ would cross zero at $Q^{2} \approx 7.7$ $(\mathrm{GeV} / \mathrm{c})^{2}$. To investigate this possibility an extension [46] of this experiment to a $Q^{2}$-value of $9.6(\mathrm{GeV} / \mathrm{c})^{2}$ has been approved to run in Hall C.

Last year an experiment [47] was completed which used the Rosenbluth technique with a number of improvements. In stead of detecting the scattered electron, the recoil proton was detected and both HRS spectrometers in Hall A were used. Significantly improved systematic errors are expected at $Q^{2}$-values up to $4(\mathrm{GeV} / \mathrm{c})^{2}$. Recently several studies have investigated the two-photon contribution to the Rosenbluth technique. Blunden et al. [48] calculated the elastic contribution to the so-called box diagram in the radiative correction and showed that this accounted for half the discrepancy between the results from the Rosenbluth technique and those from polarization transfer. The inelastic contribution is much harder to calculate but an estimate might be obtained from the transverse beam asymmetry in elastic-proton scattering. Guichon and Vanderhaeghen [4] showed in general that a two-photon exchange contribution needed to explain the discrepancy is of the order of a few percent. However, Rekalo and Tomasi-Gustafsson [50] showed, also on general principles, that the contribution from two-photon exchange should be of order $\alpha^{2}$ and thus very small. 

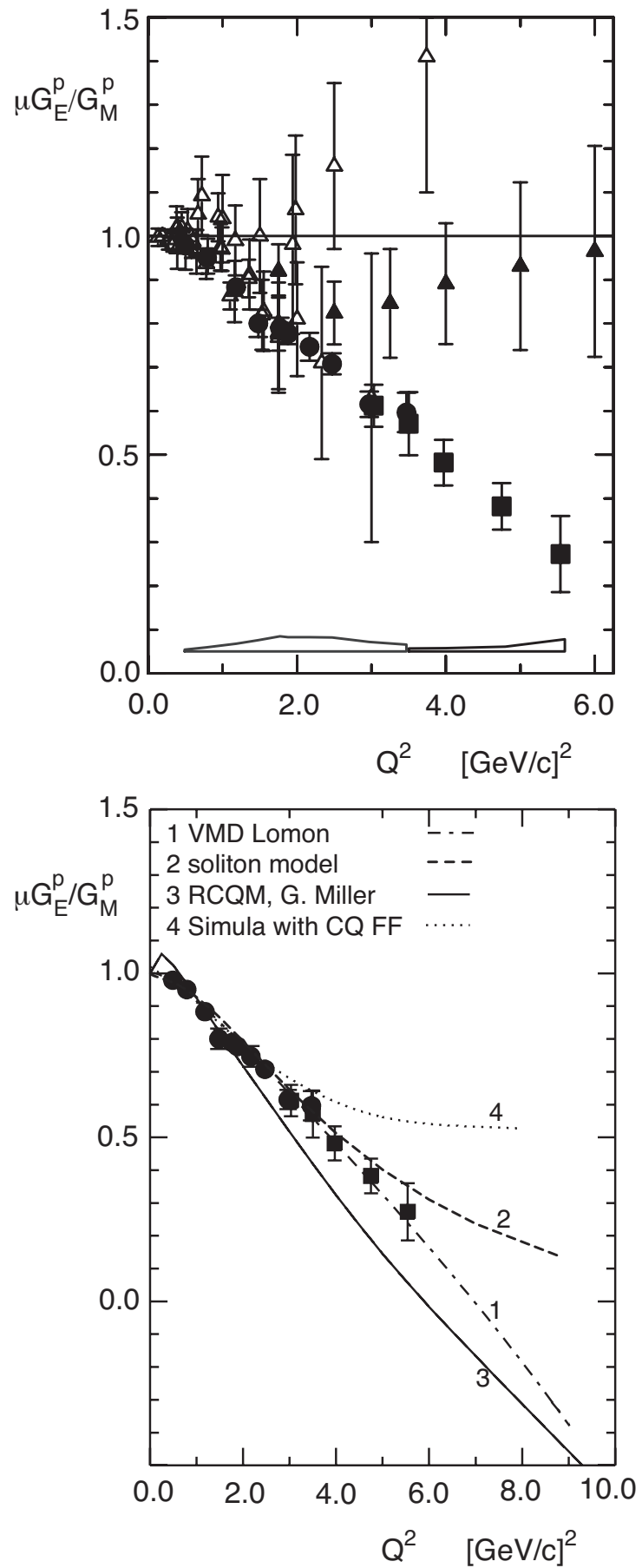

Fig. 3. The ratio $\mu G_{E}^{p} / G_{M}^{p}$ from 42 (solid circles) and 43 . (solid squares), compared to the older SLAC data (solid triangles 44]) (top) and various theoretical calculations (bottom): dash-dotted [15, dashed [20, solid 17] and dotted 19]. The systematic errors for both recent experiments are shown as a band at the bottom of the left figure

\section{Wide-angle compton scattering}

Until fairly recently, little was known about the response of a nucleon to real photons in the regime where its response is dominated by the internal structure of the nucleon. Only one twenty-year old experiment from Cornell
[51] had provided data on elastic photon scattering (Real Compton Scattering) at large transverse momentum. Two different reaction mechanisms are expected to describe Real Compton Scattering. At very large momentum transfer two-gluon exchange, in the perturbative QCD framework, will dominate the reaction. Indeed, the Cornell data exhibit an $s$ and $t$ dependence in fair agreement with the pQCD counting rule: $d \sigma / d t=f\left(\theta_{C M}\right) \cdot s^{-n}$ with $n=6$ for RCS.

Recent calculations by Radyushkin 52 have shown that at moderate momentum transfer a softer mechanism should dominate at large center-of-mass angles (WideAngle Compton Scattering, WACS). In this handbag mechanism only one quark interacts with the real photon and no gluon is exchanged between the struck quark and the rest of the partons. By factorizing the mechanism into a hard and a soft part the RCS reaction can be described in the framework of Generalized Parton Distributions (GPD) by two form factors $R_{V}$ and $R_{A}$. In the WACS regime $R_{V}$ dominates the cross section and the ratio $d \sigma_{R C S} / d \sigma_{K N}$ should be nearly independent of $s$ at a fixed value of $t$. Observation of this would be a clear indication of factorization. With a circularly polarized photon beam a measurement of the longitudinal polarization transfer coefficient $K_{L L}$ can provide a sensitive probe of the dominant reaction mechanism.

Experiment E99-114 [53] measured the RCS cross section for $s$ between 5 and $11(\mathrm{GeV} / \mathrm{c})^{2}$ and for $t$ between 1.5 and $6.5(\mathrm{GeV} / \mathrm{c})^{2}$ and $K_{L L}$ at $s=7(\mathrm{GeV} / \mathrm{c})^{2}$ and $t=$ $4(\mathrm{GeV} / \mathrm{c})^{2}$. The experiment, performed in Hall A at Jefferson Lab, used a mixed bremsstrahlung/electron beam, a liquid hydrogen target, one of the HRS spectrometers for proton detection and a lead-glass calorimeter with a sweep magnet for photon detection. The photon beam intensity was $10^{13}$ equivalent quanta per second, about 1000 times higher than in the Cornell experiment. Most of the background was eliminated by requiring an photon-proton coincidence and RCS events were separated from pion photo-production and elastic electron scattering through kinematical correlations between the energy and the angle of the detected photon and the recoiling proton.

The preliminary value for $K_{L L}$ is shown in Fig. पf from which a clear preference for the hand-bag mechanism is evident at the kinematics of the experiment. Similarly, preliminary data for the cross section follow the scaling power predicted by Radyushkin. The data of E99-114 have demonstrated that accurate RCS data can be extracted with modern photon facilities which provide sensitive information on the internal structure of the nucleon.

\section{Neutron spin structure}

\subsection{The $Q^{2}$-evolution of the Gerasimov-Drell-Hearn sum rule}

Over the past twenty years measurements of the nucleon spin structure have shown that only a small fraction (less than 20\%) of the nucleon spin can be accounted for by the spin of the quarks. Also the Bjorken sum rule has 


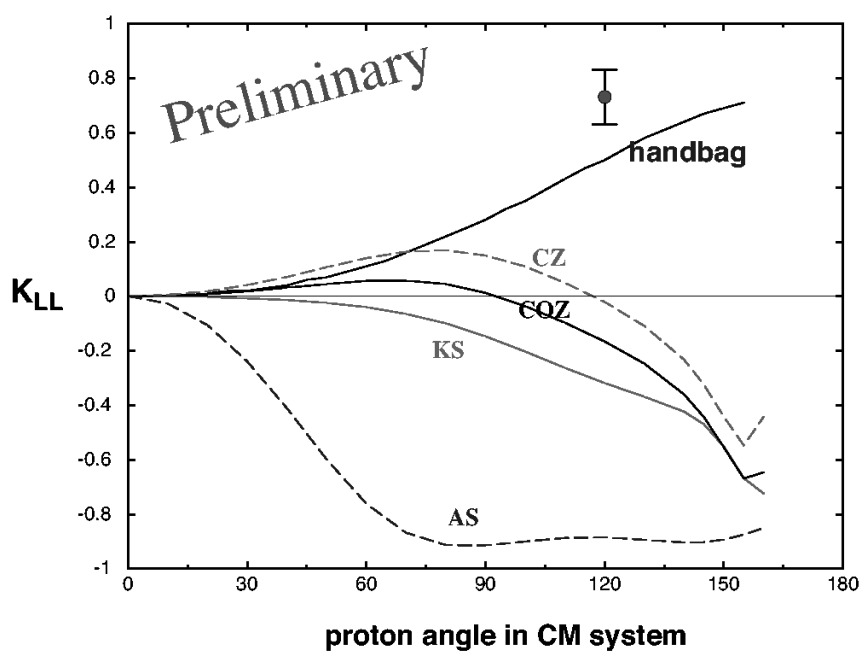

Fig. 4. The preliminary result for the polarization transfer coefficient $K_{L L}$, compared to a variety of predictions: handbag [52] and pQCD (AS asymptotic, KS King-Sachrajda, COZ Chernyak-Ogloblin-Zhitnitsky and CZ Chernyak-Zhinitsky).

been validated to within $10 \%$. Recently, the Bjorken and the Gerasimov-Drell-Hearn (GDH) sum rules have been generalized [54 over the full range of momentum transfer from the non-perturbative $Q^{2}=0$ to the perturbative $Q^{2}=$ inf limit.

This generalized GDH sum rule $I\left(Q^{2}\right)$ for the neutron has recently been studied [55] from $Q^{2}$ is 0.1 to 0.9 $(\mathrm{GeV} / \mathrm{c})^{2}$ by measuring inclusive scattering of longitudinally polarized electrons from a polarized ${ }^{3} \mathrm{He}$ target in Hall A. The polarized ${ }^{3} \mathrm{He}$ target used the principles of optical pumping of a small admixture of $\mathrm{Rb}$ and spin exchange between the Rb and the ${ }^{3} \mathrm{He}$ atoms. The scattered electrons were detected in either of the two Hall A HRS spectrometers. $\mathrm{A} \mathrm{CO}_{2}$ gas Čerenkov detector and a leadglass shower counter were used to separate electrons from the pion background. The results were corrected for the fact that the neutron was embedded in a ${ }^{3} \mathrm{He}$ nucleus by using a calculation by Ciofi degli Atti and Scopetta 56]. The results show a smooth variation of $I\left(Q^{2}\right)$ to increasing negative values as $Q^{2}$ drops from 0.9 to $0.1(\mathrm{GeV} / \mathrm{c})^{2}$. A recently completed new experiment [57] will extend the data down to $0.02(\mathrm{GeV} / \mathrm{c})^{2}$ and establish whether $I\left(Q^{2}\right)$ indeed turns upwards towards the value of the GDH integral.

In both of these experiments measurements were made with the ${ }^{3} \mathrm{He}$ target polarization oriented longitudinal and transverse to the momentum transfer, which allowed the extraction of both spin structure functions $g_{1}$ and $g_{2}$. The results from the first experiment [55] were used to study two further spin structure moments, $d_{2}$ and the Burkhardt-Cottingham sum rule [59]:

$$
\begin{array}{r}
d_{2}\left(Q^{2}\right)=\int_{0}^{1} x^{2}\left[2 g_{1}\left(x, Q^{2}\right)+3 g_{2}\left(x, Q^{2}\right)\right] d x \\
\Gamma_{2}\left(Q^{2}\right)=\int_{0}^{1} g_{2}\left(x, Q^{2}\right) d x=0
\end{array}
$$
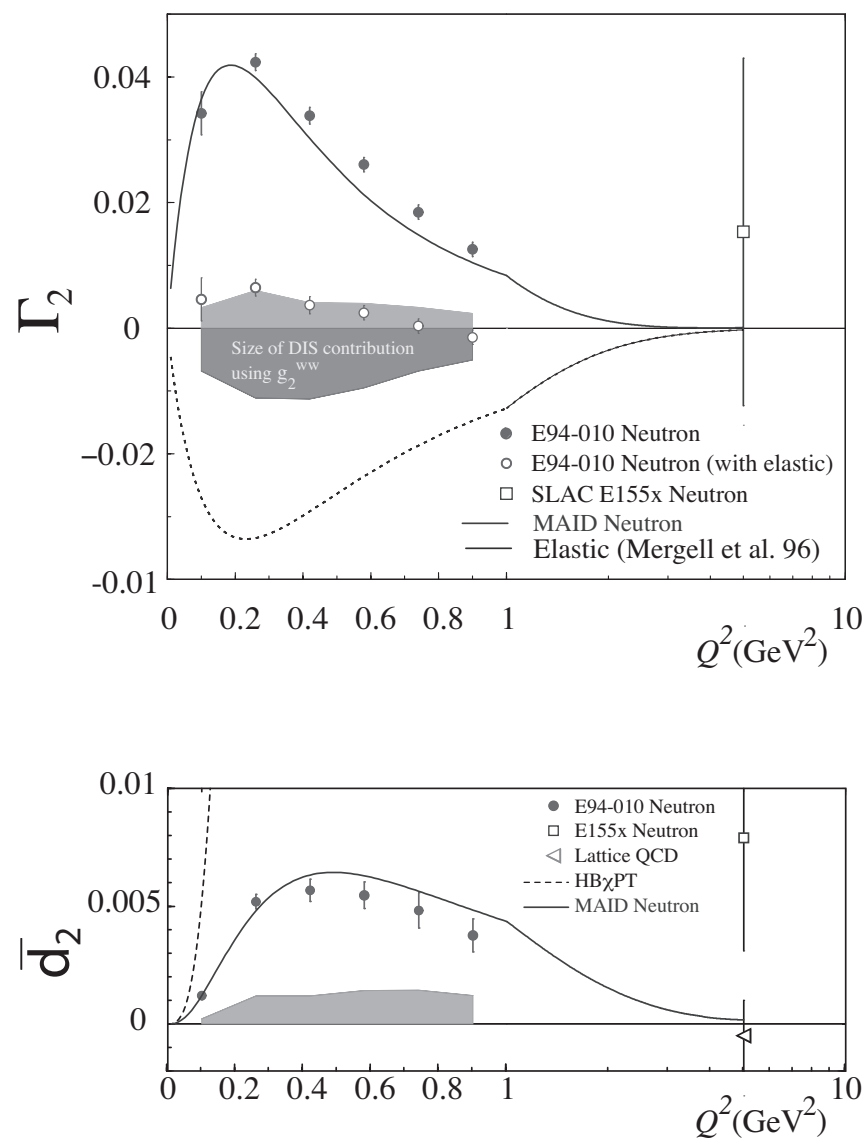

Fig. 5. Results for the Burkhardt-Cottingham sum rule $\Gamma_{2}^{n}$ (top panel) and for $\overline{d_{2}^{n}}$ (bottom panel) along with world data from DIS and theoretical predictions (see text)

The values of $g_{2}\left(x, Q^{2}\right)$ were evaluated from the experimental results, measured at fixed incident beam energies and scattering angles, by interpolation and (for a few points) extrapolation for each of six values of $Q^{2}$. The integrals were computed using limits of integration extending from the nucleon pion threshold to a value of $x$ corresponding to an invariant mass $W=2.0 \mathrm{GeV}$. Effects of the nuclear medium were again corrected for using the prescription of Ciofi degli Atti and Scopetta. The final results 58 are shown in Fig. 5 .

In the top panel $\Gamma_{2}^{n}$ is indicated by solid circles, the results after addition of the elastic contribution by open circles. The positive light band is an indication of the systematic error and the dark negative band of the estimated DIS contribution. The results clearly indicate that the Burkhardt-Cottingham sum rule is verified within uncertainties over the $Q^{2}$-range of the experiment. The open square shows the results of SLAC experiment E155x which had a significantly larger error bar. The solid curve represents the resonance contribution evaluated with the MAID code.

In the bottom panel the results for $\bar{d}_{2}\left(Q^{2}\right)=d_{2}\left(Q^{2}\right)-$ $d_{2}^{\text {elastic }}\left(Q^{2}\right)$ are shown as solid circles at the six $Q^{2}$-values. The grey band represents their systematic uncertainty. The SLAC E155x result 60 is again shown as an open square, the prediction from Lattice QCD 61] by the open 
left-pointing triangle. The solid line is the MAID calculation 62 containing only the resonance contribution. Finally, a Heavy-Baryon Chiral Perturbation Theory prediction 63 is indicated by a dashed line. At moderate $Q^{2}$ the JLab data show a positive value of $\bar{d}_{2}^{n}$, slowly decreasing with $Q^{2}$. The lattice prediction is negative, but close to zero. The precise new data will allow the extraction of twist- 3 and twist- 4 matrix elements, which contain non-trivial effects of quark-gluon interactions.

\subsection{Neutron spin structure at large $x_{B}$}

The small contribution to the nucleon spin from the quark spin, mentioned in the previous section, implies that at least one other degree of freedom is required. One possibility would be the parton orbital angular momentum (OAM). Several recent experimental results, such as on elastic electron scattering and deuteron photo-disintegration, have provided indications of the role of OAM.

A recent experiment [64] performed in Hall A at Jefferson Lab measured inclusive scattering of polarized electrons off a polarized ${ }^{3} \mathrm{He}$ target in a kinematic region where the Bjorken scaling variable $x$ is large. In this region the valence quarks dominate and the neutron spin asymmetry $A_{1}^{n}$ (the ration of the polarized and the unpolarized structure functions $g_{1} / F_{1}$ ) can be estimated based on our knowledge of the interaction between quarks. Thus, data in this domain can potentially shed light on the role of OAM of the valence quarks. This asymmetry is predicted to approach 1 as $x \rightarrow 1$. Sofar, all existent data on $A_{1}^{n}$ are either negative or consistent with zero.

Data were collected at three $x$-values, $0.33,0.47$ and 0.60 at $Q^{2}$-values ranging from 2.7 to $4.8(\mathrm{GeV} / \mathrm{c})^{2}$. The invariant mass was well above the resonance region. The beam asymmetry was measured with the target polarization oriented both parallel and transverse to the beam polarization. A model 65 for ${ }^{3} \mathrm{He}$ which includes $S, S^{\prime}$ and $D$ states and a pre-existing $\Delta(1232)$ component, was used to extract $A_{1}^{n}$ from $A_{1}^{3} \mathrm{He}$.

The results for $A_{1}^{n}$ are shown in Fig. [6 The new datum at $x=0.33$ is in good agreement with the world data, while for $x>0.4$ the precision of the new data shows an order of magnitude improvement. The new data for the first time prove that $A_{1}^{n}$ becomes positive at large $x$ and their trend is consistent with models which predict that $A_{1}^{n}$ approaches unity as $x \rightarrow 1$. None of the new data agrees with calculations which assume the BBS 66] parametrization. In this pQCD parametrization zero quark OAM and helicity conservation is assumed, which implies that a quark with $x \rightarrow 1$ must have the same helicity as the nucleon itself (hadron helicity conservation, HHC). On the other hand, the data do agree with predictions which do not assume zero OAM.

One can extract the polarized valence quark distributions $(\Delta u+\Delta \bar{u}) /(u+\bar{u})$ and $(\Delta d+\Delta \bar{d}) /(d+\bar{d})$ from $A_{1}^{n}$ by using the world data set on the unpolarized $u$ and $d$ distributions, if one assumes that in the region $x>0.3$ valence quarks truly dominate so that all strange quark distributions can be neglected. The results from the Hall A ex-

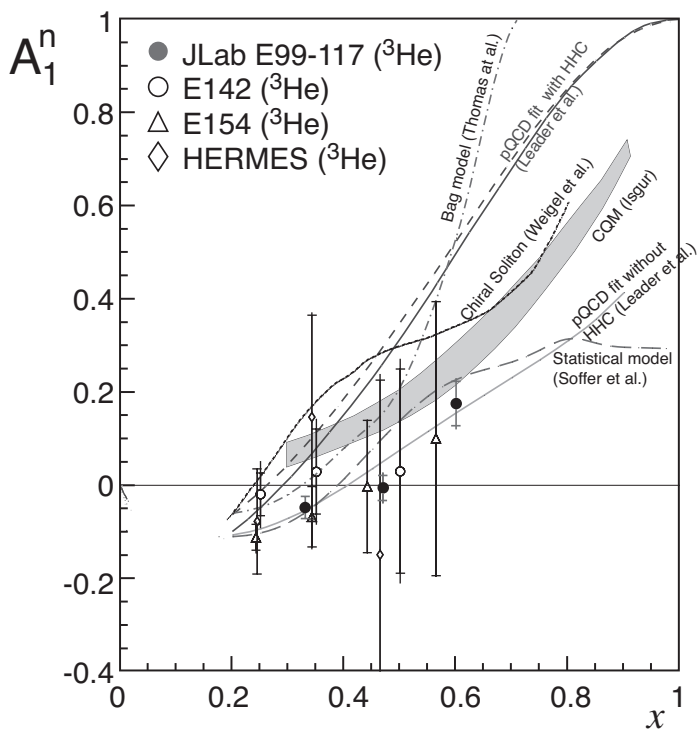

Fig. 6. Results for $A_{1}^{n}$ compared to a variety of theoretical predictions and existing data obtained with a polarized ${ }^{3} \mathrm{He}$ target

periment extend to higher $x$ and improve on the accuracy of available HERMES results 67. Again, the new data agree with e.g. the hyperfine-perturbed CQM prediction by Isgur [68, but not with that from pQCD-based HHC, which suggests that effects beyond leading-order pQCD, such as OAM, could play an important role in this kinematic region.

\section{Summary}

Recent advances in polarized electron sources, polarized nucleon targets and nucleon recoil polarimeters have made it possible to accurately probe the response of the nucleon to a variety of electromagnetic probes. New data on nucleon electro-magnetic form factors with an unprecedented precision have (and will continue to) become available in an ever increasing $Q^{2}$-domain. These data will form tight constraints on models of nucleon structure and will hopefully incite new theoretical efforts. In addition they will significantly improve the accuracy of the extraction of strange form factors from parity-violating experiments.

Acknowledgements. The author expresses his gratitude to Gerry Miller, Zein-Eddine Meziani and Bogdan Wojtsejkhowski for fruitful discussions and for receiving their results prior to publication. This work was supported by DOE contract DEAC05-84ER40150, under which the Southeastern Universities Research Association (SURA) operates the Thomas Jefferson National Accelerator Facility for the United States Department of Energy.

\section{References}

1. M.N. Rosenbluth: Phys. Rev. 79, 615 (1950)

2. P. Bosted et al.: Phys. Rev. C 51, 409 (1995) 
3. A.I. Akhiezer and M.P. Rekalo: Sov. J. Part. Nucl. 3, 277 (1974); based on J.H. Scofield: Phys. Rev. 141, 1352 (1966)

4. R. Arnold, C. Carlson and F. Gross, Phys. Rev. C 23, 363 (1981)

5. K. Aulenbacher et al.: Nucl. Instrum. Methods Phys. Res. A 391, 498 (1997)

6. M. Poelker et al.: Proc. of the 14th Int. Symp. on HighEnergy Spin Physics, Osaka (2000), p. 943

7. M. Hauger et al.: Nucl. Instrum. Methods Phys. Res. A 462, 382 (2001)

8. M. Baylac et al.: Phys. Lett. B 459, 412 (2001)

9. T.D. Averett et al.: Nucl. Instrum. Methods Phys. Res. A 427, 440 (1999)

10. P.L. Anthony et al.: Phys. Rev. Lett. 71, 959 (1993); Phys. Rev. D 54, 6620 (1996); J.S. Jensen and Ph.D. Thesis: CalTech, 2000 (unpublished)

11. R. Surkau et al.: Nucl. Instrum. Methods Phys. Res. A 384, 444 (1997)

12. L. Bimbot et al.: submitted to Nucl. Instrum. Methods Phys. Res. A

13. M. Ostrick et al.: Phys. Rev. Lett. 83, 276 (1999)

14. G. Höhler et al.: Nucl. Phys. B 114, 505 (1976)

15. E.L. Lomon: Phys. Rev. C 64, 035204 (2001)

16. F. Schlumpf: J. Phys. G 20, 237 (1994)

17. M.R. Franck, B.K. Jennings, and G.A. Miller: Phys. Rev. C 54, 920 (1995)

18. J. Ralston et al.: Proc. 7th CIPANP, Quebec City (2000), p. 302

19. F. Cardarelli and S. Simula: Phys. Rev. C 62, 065201 (2000)

20. G. Holzwarth: Z. Phys. A 356, 339 (1996)

21. H. Gao et al.: Phys. Rev. C 50, R546 (1994)

22. P. Markowitz et al.: Phys. Rev. C 48, R5 (1993)

23. E.E.W. Bruins et al.: Phys. Rev. Lett. 75, 21 (1995)

24. H. Anklin et al.: Phys. Lett. B 336, 313 (1994)

25. H. Anklin et al.: Phys. Lett. B 428, 248 (1998)

26. G. Kubon et al.: Phys. Lett. B 524, 26 (2002)

27. W. Xu et al.: Phys. Rev. Lett. 85, 2900 (2000)

28. W. Xu et al.: Phys. Rev. C 67, 012201 (2003)

29. W. Brooks and M.F. Vineyard: JLab experiment E94-017

30. T. Eden et al.: Phys. Rev. C 50, R1749 (1994)

31. M. Meyerhoff et al.: Phys. Lett. B 327, 201 (1994)

32. I. Passchier et al.: Phys. Rev. Lett. 82, 4988 (1999)

33. C. Herberg et al.: Eur. Phys. Jour. A 5, 131 (1999)
34. J. Becker et al.: Eur. Phys. Jour. A 6, 329 (1999)

35. D. Rohe et al.: Phys. Rev. Lett. 83, 4257 (1999)

36. S. Galster et al.: Nucl. Phys. B 32, 221 (1971)

37. H. Schmieden et al.: MAMI proposal A1/2-99

38. H. Zhu et al.: Phys. Rev. Lett. 87, 081801 (2001)

39. R. Madey et al.: Phys. Rev. Lett. 91, 122002 (2003)

40. Bates Large Acceptance Spectrometer Toroid: http://mitbates.mit.edu /blast

41. B. Wojtsekhowski et al.: JLab experiment E02-013

42. M.K. Jones et al.: Phys. Rev. Lett. 84, 1398 (2000)

43. O. Gayou et al.: Phys. Rev. Lett. 88, 092301 (2002)

44. L. Andivahis: Phys. Rev. D 50, 5491 (1994) and references therein

45. S.J. Brodsky and G. Farrar: Phys. Rev. D 11, 1309 (1975)

46. C.F. Perdrisat et al.: JLab experiment E01-109

47. J. Arrington and R. Segel: JLab experiment E01-001

48. P.G. Blunden, W. Melnitchouk, and J.A. Tjon: nuclth/0306076

49. P.A.M. Guichon and M. Vanderhaeghen: hep-ph/0306007

50. M.P. Rekalo and E. Tomasi-Gustafsson: nucl-th/0307066

51. M.A. Shupe et al.: Phys. Rev. D 19, 1929 (1979)

52. A.V. Radyushkin: Phys. Rev. D 56, 5524 (1997)

53. C. Hyde-Wright, A. Nathan, and B. Wojtsekhowski: Jefferson experiment E99-114

54. X. Ji and J. Osborne: J. Phys. G 27, 127 (2001)

55. M. Amarian et al.: Phys. Rev. Lett. 89, 241301 (2002)

56. C. Ciofi degli Atti and S. Scopetta: Phys. Lett. B 404, 223 (1997)

57. J.-P. Chen, A. Deur, and F. Garibaldi: Jefferson experiment E97-110

58. M. Amarian et al.: submitted to Phys. Rev. Lett.

59. H. Burkhardt and W.N. Cottingham: Ann. Phys. 56, 453 (1970)

60. P.L. Anthony et al.: Phys. Lett. B 553, 18 (2003)

61. M. Göckeler et al.: Phys. Rev. D 63, 074506 (2001)

62. D. Drechsel, S. Kamalov, and L. Tiator: Phys. Rev. D 63, $114010(2001)$

63. C.W. Kao, T. Spitzenberg, and M. Vanderhaeghen: Phys. Rev. D 89, 016001 (2003)

64. X. Zheng et al.: submitted to Phys. Rev. Lett.

65. F. Bissey et al.: Phys. Rev. C 65, 064317 (2002)

66. S.J. Brodsky, M. Burkardt, and I. Schmidt: Nucl. Phys. B 441, 197 (1995)

67. K. Ackerstaff et al.: Phys. Lett. B 464, 123 (1999)

68. N. Isgur: Phys. Rev. D 59, 034013 (1999) 